Hou Wenhao (Orcid ID: 0000-0002-7220-5721)

Azadifar Mohammad (Orcid ID: 0000-0002-0489-1838)

Rubinstein Marcos (Orcid ID: 0000-0003-4806-038X)

Rachidi Farhad (Orcid ID: 0000-0002-2169-9549)

Zhang Qilin (Orcid ID: 0000-0002-6641-0251)

\title{
The polarity reversal of lightning-generated sky wave
}

\section{W. Hou ${ }^{1,2}$, M. Azadifar ${ }^{3}$, M. Rubinstein ${ }^{3}$, F. Rachidi ${ }^{2}$, and Q. Zhang ${ }^{1}$}

${ }^{1}$ Key Laboratory of Meteorological Disaster, Ministry of Education (KLME)/Collaborative Innovation Center on Forecast and Evaluation of Meteorological Disaster (CIC-FEMD)/Key Laboratory for Aerosol-Cloud-Precipitation of China Meteorological Administration, Nanjing University of Information Science and Technology, Nanjing 210044, China.

${ }^{2}$ Electromagnetic Compatibility Laboratory, Swiss Federal Institute of Technology, Lausanne 1015, Switzerland.

${ }^{3}$ School of Management and Engineering Vaud, University of Applied Sciences and Arts Western Switzerland, Yverdon-les-Bains 1400, Switzerland.

Corresponding author 1: Qilin Zhang (zhangqilin71@163.com)

Corresponding author 2: Farhad Rachidi (farhad.rachidi@epfl.ch)

\section{Key Points:}

- A method based on the FDTD method and the EM superposition principle is proposed to study the reflection of LEMP by the lower ionosphere

- The dominance of radiation from the horizontal or vertical equivalent current in Fresnel zone determines the polarity of the first skywave

- The enhancement of the horizontal current density at $~ 90 \mathrm{~km}$ contributes to the double-peak skywave for a westward propagation

\section{Abstract}

The polarity reversal of the lightning-generated first sky wave as a function of the observation distance is studied using a novel approach combining the finite-difference timedomain (FDTD) method and the superposition principle of electromagnetic waves. In this method, the sky wave is generated by radiation from the induced current produced by the motion of charged particles driven by the lightning-radiated electromagnetic waves in the ionosphere. The horizontal and vertical components of the induced current density under the daytime and nighttime ionospheric conditions are evaluated. Their different contributions to the sky wave at different observation distances are analyzed in detail. Furthermore, a physical explanation for the polarity reversal in the time domain is proposed. It is found that, for relatively short observation distances (within $\sim 200 \mathrm{~km}$ ), the first sky wave is dominated by the component generated by the horizontal equivalent current in the Fresnel zone, while for

This article has been accepted for publication and undergone full peer review but has not been through the copyediting, typesetting, pagination and proofreading process which may lead to differences between this version and the Version of Record. Please cite this article as doi: 10.1029/2020JD032448 
longer observation distances (larger than $\sim 300 \mathrm{~km}$ ), the first sky wave is dominated by the component generated by the vertical equivalent current in the Fresnel zone. Since the polarities of the sky wave components generated by the vertical current source and horizontal current source are opposite, the polarity of the sky wave will reverse when increasing the observation distance.

\section{Introduction}

The lightning-radiated electromagnetic pulse (LEMP) can be reflected by the lower ionosphere (D region) and can propagate hundreds to thousands of kilometers in the form of sky waves in the Earth-ionosphere waveguide (EIWG). The observed electromagnetic (EM) fields at distant observation points contain therefore information on the source and on the propagation path. Numerous models have been established to study the propagation of LEMP in the EIWG, to infer the lightning current moment (e.g., Cummer \& Inan, 2000; Li et al., 2008) or the electron density profile in the ionosphere (e.g., Cummer et al., 1998; Cheng \& Cummer, 2005; Han \& Cummer, 2010a, b; Shao et al., 2013). These models are mainly based either on mode theory (e.g., Budden, 1988, Ferguson et al., 1989), ray theory (e.g., Jacobson et al., 2009; Qin et al., 2017), or the finite-difference time-domain method (e.g., Cummer et al., 1998; Berenger, 2005; Hu \& Cummer, 2006; Marshall, 2012; Tran et al., 2017). Several characteristics of the sky wave in the frequency domain and in the time domain have also been obtained adopting these models.

Qin et al. (2017) proposed an improved ray theory and transfer matrix method-based model to study the propagation of LEMP in the EIWG. Using this model, they studied the sky wave characteristics of several lightning sferics observed in Central China. Specifically, they examined the dependence of the waveform on the propagation directions in a frequency band ranging from 3 to $150 \mathrm{kHz}$. Their results show that, for an eastward propagation of the EM wave under nighttime conditions, the sky wave would be characterized by a double-peak waveform. They attributed this feature to the magneto-ionic splitting phenomenon in the lower ionosphere due to the Earth's magnetic field. On the other hand, the influence of the geomagnetic field on the sky wave was found to be less important during daytime.

Tran et al. (2017) studied the lightning's full-bandwidth electromagnetic fields on the ground surface at observation distances ranging from 50 to $500 \mathrm{~km}$ without considering the geomagnetic field. They used a realistic return-stroke representation in their model and examined, for the first time, the influence of the lightning's source parameters on the sky wave waveforms. They also compared the ionosphere reflection heights estimated using the time intervals between the positive ground wave and the positive and negative parts of the first sky wave. Their results showed that the mismatch between the estimated reflection height and the reference height was less when using the negative peak of the first sky wave. Therefore, they speculated that the negative part of the sky wave was due to the radiation component and the positive part was due to the induction component of the electric field.

In the above simulation studies, an interesting characteristic of the first sky wave was that its initial polarity was the same as that of the ground wave at relatively close observation distances, reversing at larger observation distances, regardless of whether or not the geomagnetic field was considered. The reversal distances were found to be about $200 \mathrm{~km}$ and $300 \mathrm{~km}$ for daytime and nighttime ionosphere conditions, respectively (Tran et al., 2017). Haddad et al. (2012) analyzed the wideband (from $16 \mathrm{~Hz}$ to at least $10 \mathrm{MHz}$ ) vertical electric field of more than 500 first and subsequent return strokes acquired at observation distances ranging from 10 to $330 \mathrm{~km}$ under daytime conditions. Somu et al. (2015) presented 
additional data acquired under nighttime conditions. Azadifar et al. (2017) and Mostajabi et al. (2019) presented the simultaneously recorded current and electric field at $380 \mathrm{~km}$ associated with a lightning strike to the Säntis Tower. The data presented in these studies also show the polarity reversal of the first sky wave. Smith et al. (2004) analyzed the vertical electric field radiated by narrow bipolar pulses (NBPs) recorded by the Los Alamos Sferic Array (LASA). They found that the leading-edge polarities of the reflections would reverse at distance ranges of 150 to $200 \mathrm{~km}$. The polarity reversal was attributed to quasi-Brewster angle effects.

In fact, the polarity reversal in the time domain corresponds to the sign change (or phase change) of the complex refection coefficients in the frequency domain from vertical incidence to grazing incidence, as discussed by Wait and Walters (1963), Volland (1995), Smith et al. (2004) and Said (2009).

In this paper, we propose another physical explanation in the time domain for the polarity reversal of the lightning-generated first sky wave, using an approach that combines the finite-difference time-domain (FDTD) method and the superposition principle of EM waves. We first analyze the lightning-generated induced current in the ionosphere calculated by the FDTD method. The current in the ionosphere is then taken as a new source and used to simulate the sky waves at observation distances ranging from 100 to $400 \mathrm{~km}$. Firstly, we study the sky waves under typical daytime ionosphere conditions without considering the geomagnetic field. The horizontal and vertical current density components in the ionosphere are evaluated and their different contributions to the sky waves at different observation distances are analyzed. The analysis provides a physical explanation for the polarity reversal of the sky wave in the time domain. Furthermore, we test the validity of the explanation under nighttime ionospheric conditions, in which the lower ionosphere is treated as a magnetized and anisotropic plasma. The results and analysis presented in the following sections will provide new insights into the understanding of the sky-wave waveform characteristics.

\section{Methodology}

\subsection{FDTD method for LEMP propagation}

The lower ionosphere can be regarded as a cold magnetized plasma, which is full with electrons, negative ions and positive ions. The coupling of the lightning electromagnetic (EM) wave with these charged particles can be described by Maxwell's equations along with the Langevin equation, which can be expressed as (e.g., Hu \& Cummer, 2006; Marshall, 2012)

$$
\nabla \times \mathbf{E}=-\mu_{0} \frac{\partial \mathbf{H}}{\partial t}
$$

$$
\begin{gathered}
\nabla \times \mathbf{H}=\varepsilon_{0} \frac{\partial \mathbf{E}}{\partial t}+\mathbf{J}_{t o t}+\mathbf{J}_{s} \\
\frac{\partial \mathbf{J}_{n}}{\partial t}+v_{n} \mathbf{J}_{n}=\frac{q_{n}}{\left|q_{n}\right|} \omega_{B, n}\left(\mathbf{J}_{n} \times \mathbf{b}_{E}\right)+\varepsilon_{0} \omega_{p, n}^{2} \mathbf{E}
\end{gathered}
$$

where

- $n$ represents the three types of particles, 
- $\omega_{p, n}=\sqrt{q_{n}^{2} N_{n} / \varepsilon_{0} m_{n}}$ is the plasma frequency, $q_{n}, m_{n}$ and $N_{n}$ being, respectively, the charge, mass and density of species $n$,

- $\omega_{B, n}=\left|q_{n} \mathbf{B}_{\mathbf{E}}\right| / m_{n}$ is the gyrofrequency, $\mathbf{B}_{\mathbf{E}}$ being the vector geomagnetic field,

- $\mathbf{b}_{E}$ is the unit vector in the direction of the geomagnetic field,

- $v_{n}$ is the collision frequency with neutral air,

$-\mathbf{J}_{\text {tot }}=\sum_{n} \mathbf{J}_{n}$ is the total current produced by each charge species, and

- $\mathbf{J}_{s}$ is the lightning current source.

The two-dimensional cylindrical finite-difference time-domain (FDTD) model proposed by Hu and Cummer (2006) was used in the present study to simulate the propagation of the lightning-radiated EM wave in the Earth-ionosphere waveguide (EIWG) and to calculate the lightning-generated current in the ionosphere. A brief description of the model will be presented in what follows. Figure 1(a) shows the geometry of the FDTD model. The upper and right boundaries are surrounded by nearly perfectly matched layer (NPML) absorbing boundaries (Cummer, 2003). The surface impedance boundary condition (SIBC) is applied to the bottom boundary to include the propagation effect (Maloney \& Smith, 1992). The observation point is located at ground level at a horizontal distance $d$ from the lightning channel. Note that the positive direction of vectors is also shown in Figure 1(a). The $E-J$ method (Cummer, 1997), which collocates $\mathbf{E}$ and $\mathbf{J}$ in time and space is used (see Figure 1(b)), since the stability condition for the magnetized plasma is the same as that in free space when adopting this method. The components of the field and current density are updated using matrix operations at each time step. In addition, in order to avoid stability and accuracy problems, scaled values for $\mathbf{H}$ and $\mathbf{J}$ are used in each iteration. The Earth's curvature can also be considered by modifying the refractive index at different heights. More details on this model can be found in $\mathrm{Hu}$ and Cummer (2006). In our simulation, the size of the FDTD simulation domain is $410 \mathrm{~km} \times 110 \mathrm{~km}$. The grid size and the time step were set, respectively, to $\Delta r=\Delta z=250 \mathrm{~m}$ and $\Delta t=0.5 \mu \mathrm{s}$.

The electron density in the lower ionosphere is modeled by a two-parameter exponential formulation (e.g., Bickel at al., 1970, Thomson, 1993),

$$
N_{e}(h)=1.43 \times 10^{13} e^{-0.15 h^{\prime}} e^{(\beta-0.15)\left(h-h^{\prime}\right)}\left(\mathrm{m}^{-3}\right)
$$

where $h$ is the height above the ground in $\mathrm{km}, h^{\prime}$ is the reference height of the ionosphere in $\mathrm{km}$, and $\beta$ is a constant in $\mathrm{km}^{-1}$. For the daytime ionosphere condition, $h$ ' is set to $70 \mathrm{~km}$ and $\beta$ is set to $0.3 \mathrm{~km}^{-1}$; for the nighttime condition, $h$ ' is set to $85 \mathrm{~km}$ and $\beta$ is set to $0.5 \mathrm{~km}^{-1}$ (Han \& Cummer, 2010a, b). The density of positive ions is set to the same value as the electrons but with a minimum value of $200 \mathrm{~cm}^{-3}$ for the daytime condition and $100 \mathrm{~cm}^{-3}$ for the nighttime condition. The collision frequencies of the electrons $\left(v_{e}\right)$ and ions $\left(v_{i}\right)$ are expressed as follows (Wait \& Spies, 1964; Morfitt \& Shellman, 1976; Cummer et al., 1998)

$$
\begin{gathered}
v_{e}(h)=1.816 \times 10^{11} e^{-0.15 h}\left(\mathrm{~s}^{-1}\right) \\
v_{i}(h)=4.54 \times 10^{9} e^{-0.15 h}\left(\mathrm{~s}^{-1}\right)
\end{gathered}
$$

The lightning channel was modeled as a dipole with a height of $10 \mathrm{~km}$, and the return stroke current moment is given by (e.g., Hu \& Cummer, 2006; Cummer et al., 1998) 


$$
I_{m}(t)=I_{0} \frac{v_{0}}{\gamma}\left[e^{-a t}-e^{-b t}\right]\left[1-e^{-\gamma t}\right]
$$

where $I_{0}=20 \mathrm{kA}, v_{0}=8 \times 10^{7} \mathrm{~m} / \mathrm{s}, \gamma=3 \times 10^{4} \mathrm{~s}^{-1}, a=2 \times 10^{4} \mathrm{~s}^{-1}$, and $b=2 \times 10^{5} \mathrm{~s}^{-1}$. The energy of this current source is mainly distributed in the VLF range. The current is assumed to be uniform along the lightning discharge channel, and it can be obtained by dividing the current moment by the channel length. Figure 2 shows the current waveform along the channel.

Our FDTD codes were validated using the measured vertical electric field data presented by Qin et al. (2017), see Appendix A for details.

\subsection{A new method for the computation of the sky wave}

The FDTD method described in the previous subsection allows us to simulate the propagation of lightning-radiated EM waves within the Earth-ionosphere waveguide. For an arbitrary observation point on the ground surface, the ground wave or the full wave composed by the ground wave and the sky wave can be easily obtained using the FDTD method. Then, the sky wave can be calculated by subtracting the ground wave from the complete waveform. In what follows, we will refer to this method as the classical method.

In this section, we will propose another method to calculate the sky wave. The lightning-radiated EM wave propagates into the ionosphere, and the charged particles (especially the electrons) in the ionosphere will be accelerated by the electric field force. The electrons collide with neutral molecules and the kinetic energy from the collision is converted to molecules' internal energy. The motion of charged particles also produces an induced current in the ionosphere, which is expressed by $\boldsymbol{J}_{\text {tot }}$ in equations (2)-(3). These current sources radiate in turn EM waves in space. The observation point on the ground surface is in the region without sources. According to the superposition principle, the EM field at the observation point is composed of the EM field produced by the lightning current, by the current in the ionosphere, and by their image currents in the ground. The EM field produced by the lightning current and its image current corresponds to the ground wave, while the EM field produced by the current in the ionosphere and its image current corresponds to the sky wave. According to this analysis, we propose a method based on the superposition principle to calculate the sky wave. This method is divided into two steps:

(1) The lightning-generated current density in the ionosphere is computed using the FDTD method, and the resulting current-density distribution is stored at each time step.

(2) The lightning source is removed from the FDTD method and the ionosphere current density at each time step is loaded as the source in the FDTD method. The resulting field waveform is computed at the observation point.

$J_{t o t}$ is the effective current density at the local position of the ionosphere. It is a threedimensional vector. If the Earth's geomagnetic field is not considered, it only contains the horizontal $\left(\vec{a}_{r}\right)$ and vertical $\left(\vec{a}_{z}\right)$ components. In the presence of the geomagnetic field, it also contains a component in the direction of $\vec{a}_{\varphi}$. However, the component in the direction of $\vec{a}_{\varphi}$ contributes little to the vertical electric field on the ground (this can be readily confirmed by assuming the current sources in the direction of $\vec{a}_{\varphi}$ as small dipoles). Therefore, only the horizontal $\left(\vec{a}_{r}\right)$ and vertical $\left(\vec{a}_{z}\right)$ components of $\boldsymbol{J}_{t o t}$ will be considered in this paper. In the second step, the horizontal and the vertical current sources can be loaded separately into the FDTD method so that the contribution of these two components to the sky wave can be examined. 
It is worth noting that, in the second step, the ionosphere should be removed and, except for the ground, the whole simulation domain should be treated as free space. If the ionosphere's charged particles were considered in the second step in addition to $\boldsymbol{J}_{t o t}$, the motion of these charged particles would produce additional current in the ionosphere, resulting a different current density compared with that calculated in the first step. We will validate this new approach by comparing its results with those obtained by the classical method.

\section{Results and analysis}

3.1 Validation of the proposed method to compute the sky wave

Figure 3 shows the comparison of sky waves calculated using the classical method and the proposed method for a typical daytime condition $\left(h^{\prime}=70 \mathrm{~km}\right.$ and $\beta=0.3 \mathrm{~km}^{-1}$ ) without considering the Earth's geomagnetic field. Figure 3(a) shows the calculated vertical electric field at an observation distance of $200 \mathrm{~km}$ from the lightning channel. In addition, the ground wave and the sky wave that are extracted from the complete waveform are also presented in the same figure. Figure 3(b) shows the sky wave components generated by the horizontal and vertical current sources in the ionosphere. The total sky wave is obtained by summing up these two components according to our proposed method. It can clearly be seen from Figure 3(c) that the calculated sky waves adopting these two methods are the same (the difference is only $0.02 \%$ ), thus validating our proposed approach based on the superposition principle.

Figure 4 further shows a comparison of the results for a typical nighttime condition ( $h^{\prime}=85 \mathrm{~km}$ and $\beta=0.5 \mathrm{~km}^{-1}$ ) considering the Earth's geomagnetic field. The lightningradiated EM wave is assumed to propagate from West to East (eastward propagation). The difference in peaks calculated by the classical method and our proposed method is about $3 \%$, which is caused by the neglect of $\vec{J}_{\varphi}$ and by numerical errors. This further validates our proposed approach as a very good approximation even in the anisotropic case.

\subsection{Ground wave}

Figure 5 shows the simulated ground wave electric field at ground level for observation distances ranging from $100 \mathrm{~km}$ to $400 \mathrm{~km}$. The polarity of the ground wave produced by a negative return stroke is negative according to the vector direction definition shown in Figure 1(a).

3.3 Sky wave under daytime ionosphere conditions

In the VLF band, the reflection of the electromagnetic (EM) wave occurs approximately at a height where (e.g., Ratcliffe, 1959; Said, 2009)

$$
\omega_{p, e}^{2}=\omega v_{e}
$$

where $\omega$ is the angular frequency of the EM waves, $\omega_{p, e}$ is the plasma frequency of electrons, and $v_{e}$ is the collision rate of electrons in Equation (5). Both $\omega_{p, e}$ and $v_{e}$ change with the height. For a typical daytime ionosphere, the reflection height is $\sim 70 \mathrm{~km}$. At such heights, the electron-neutral collision rate is much larger than the electron gyrofrequency. In this situation, we can ignore the effect of the geomagnetic field and treat the ionosphere as an isotropic medium. In addition, since the mass of the ions is much larger than that of the electron, we will only consider the motion of the electrons and ignore the effect of ions. 
Figure 6 shows the scaled induced current density $\left(J \times \Delta t / \varepsilon_{0}\right)$ at different heights $(h)$ and horizontal distances $(d)$ under typical daytime ionosphere conditions. It can clearly be seen that the initial peaks of the horizontal components of the current density are all positive and the initial peaks of the vertical components are all negative. Since the induced currents are produced by the electron motion driven by the electric field force, we can attribute the polarity of the current density to the polarity of the lightning-radiated electric field at the particular ionosphere height. For the negative return stroke case studied here, the lightningradiated electric field above the ground is composed of a positive horizontal component and a negative vertical component. As a result, the horizontal component of the current density is positive and the vertical component is negative. Note that all the polarities of the current density or the electric field described in this paper refer to the polarity of the initial peak (peak of the initial half-cycle).

Figure 7 shows the simulated sky wave at observation distances ranging from $100 \mathrm{~km}$ to $400 \mathrm{~km}$, at ground level. The red lines show the sky wave components generated by the horizontal equivalent current in the ionosphere, which are all characterized by negative initial peaks. The blue lines show the sky wave components generated by the vertical equivalent current in the ionosphere, which are all characterized by positive initial peaks. The black lines are the total sky wave. Note that the current density in the entire ionosphere was considered in this calculation. It can clearly be seen that the initial polarity of the sky wave turns from negative to positive as the observation distance increases. At an observation distance of $100 \mathrm{~km}$, the sky wave is dominated by the component generated by the horizontal equivalent current. Thus, the total vertical electric field has a negative initial peak, of the same polarity as the ground wave. However, when the observation distance is larger than about $300 \mathrm{~km}$, the sky wave is dominated by the component generated by the vertical equivalent current in the ionosphere, resulting in a positive initial peak that is opposite to the groundwave polarity.

In the following, we will present a more intuitive way to better understand the different contributions of the vertical and horizontal equivalent current.

The sky wave is radiated by the currents in the ionosphere, especially by the currents in the Fresnel zone. The range of the Fresnel zone can be estimated using the method presented by Lay and Shao (2011), which found the Fresnel zone considering the phase difference of different propagation ray paths. For a daytime ionosphere with a reflection height of $70 \mathrm{~km}$ and an electromagnetic wave at $15 \mathrm{kHz}$, the estimated largest radius of the Fresnel zone in the propagation direction $\left(\boldsymbol{a}_{\boldsymbol{r}}\right)$ is about $36 \mathrm{~km}$ for an observation distance of $100 \mathrm{~km}$, and $120 \mathrm{~km}$ for an observation distance of $400 \mathrm{~km}$. In the transverse direction $\left(\boldsymbol{a}_{\boldsymbol{\varphi}}\right)$, the estimated radii of the Fresnel zone are about $30 \mathrm{~km}$ and $45 \mathrm{~km}$ for observation distances of $100 \mathrm{~km}$ and $400 \mathrm{~km}$, respectively. In the following, we focus our analysis on the current in the vertical cross section that is along the path from the source to the observation point.

The grid size in the FDTD model is $250 \mathrm{~m}$, which is very short compared to the wavelength in the VLF band $(>10 \mathrm{~km})$. Therefore, these current sources in the ionosphere can be equivalent to Hertzian dipoles. For the problem studied here, the distances between the dipoles and observation points at ground level are several hundred kilometers, which are much larger than the wavelength in the VLF band. Therefore, we can approximately consider only the radiation term of the field (far field) generated by the dipole on the surface of a perfectly conducting ground. The vertical electric field radiated by a vertical dipole can be expressed as (Uman et al., 1975) 


$$
E_{z}^{\mathrm{v}}(r, t)=-\frac{r^{2}}{2 \pi \varepsilon_{0} c^{2} R^{3}} \frac{d I(t-R / c) \Delta z}{d t}
$$

where $I(t)$ is the equivalent current of the dipole at a considered point, $\Delta z$ is the grid size in the vertical direction (equivalent length of the dipole), $c$ is the speed of light, $\varepsilon_{0}$ is the permittivity of free space, $R$ is the distance from the dipole to the observation point, and $r$ is the horizontal distance from the dipole to the observation point. For a horizontal dipole, when the observation point is within the vertical section crossing the dipole, the radiated vertical electric field can be expressed as

$$
E_{z}^{\mathrm{h}}(r, t)=-\frac{r h}{2 \pi \varepsilon_{0} c^{2} R^{3}} \frac{d I(t-R / c) \Delta r}{d t}
$$

where $\Delta r$ is the grid size in the horizontal direction, and $h$ is the height of the dipole.

Figure 8 illustrates the contributions of a horizontal dipole and a vertical dipole located at the central point of the Fresnel zone to the sky wave observed at ground level. From the expression for the radiation component of the electric field from a small dipole, it can readily be seen that the electric field at the observation point generated by a positive horizontal current source has a negative vertical component. On the other hand, the electric field generated by a negative vertical current source has a positive vertical component. Assume the currents of these two dipoles are the same. It can be seen from figure 8(b) that, at a short observation distance, since the zenith angle for the horizontal dipole $\left(\theta_{h}\right)$ is larger than that for the vertical dipole $\left(\theta_{v}\right)$, the radiation of the horizontal dipole will be larger than that of the vertical dipole according to the radiation pattern of the dipole (see Figure $8 b$ ). For larger observation distances (see Figures 8(c) and (d)), the radiation from the vertical dipole is dominant since $\theta_{v}>\theta_{h}$.

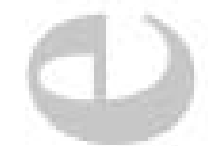

The field at the observation point is not only related to the relative position of the source and the observation point, but it is also determined by the derivative of the dipole current as indicated by equations (9) and (10). In order to comprehensively consider these factors, we define a "contribution factor" $F$ defined as the ratio of the field peaks produced by the horizontal dipole and the vertical dipole, which is expressed as follows according to equations (9) and (10)

$$
F=\frac{\left[\frac{d J_{v}(t)}{d t}\right]^{\text {peak }}}{\left[\frac{d J_{h}(t)}{d t}\right]^{\text {peak }}} \frac{r}{h}
$$

where $\left[\frac{d J_{v}(t)}{d t}\right]^{\text {peak }}$ and $\left[\frac{d J_{h}(t)}{d t}\right]^{\text {peak }}$ are the initial peaks of the current density time-derivative associated with the vertical dipole and horizontal dipole, respectively. The parameter $r$ is the lateral distance from the dipole to the observation point, and $h$ is the height of the dipole as shown in Figure 8. Let us define the first term $\left(\left[\frac{d J_{v}(t)}{d t}\right]^{\text {peak }} /\left[\frac{d J_{h}(t)}{d t}\right]^{\text {peak }}\right)$ on the right-hand side of Equation (11) as $F_{1}$ and let us call it the "peak factor". Similarly, let us define the second term $(r / h)$ as $F_{2}$ and let us call it "geometry factor". 
Figure 9 shows the calculated factors $F$ (red lines), $F_{1}$ (black lines), and $F_{2}$ (blue lines) at two heights, $65 \mathrm{~km}$ and $70 \mathrm{~km}$, both below the reflection height. Figures 9(a) and (b) are the results for the field observation points on the ground at $100 \mathrm{~km}$ and $400 \mathrm{~km}$, respectively. The shadow region marks the horizontal range of the Fresnel zone. It can be seen from Figure 9(a) that, for an observation distance of $100 \mathrm{~km}$, all the factors $F_{1}, F_{2}$ and $F$ are smaller than 1 in almost the whole Fresnel zone. This means, on the one hand, that the current derivative of the horizontal dipole is larger than that of the vertical dipole at the same position; on the other hand, even when the current moments of the horizontal dipole and the vertical dipole are the same, the field radiated by the horizontal dipole is still larger than the field radiated by the vertical dipole due to the difference in the relative position of the dipoles relative to the observation point. These are the reasons why the sky wave at short observation distances is dominated by the component produced by the horizontal current source in the Fresnel zone. For an observation distance of $400 \mathrm{~km}$ (see Figure 9(b)), although the "peak factor" $\left(F_{1}\right)$ at a height of $70 \mathrm{~km}$ is smaller than 1 , the "geometry factor" $\left(F_{2}\right)$ is much larger than 1 , thus resulting in a "contribution factor" $F$ larger than 1 over most of the Fresnel zone region. In other words, for larger observation distances, the geometry factor makes the sky wave be dominated by the component generated by the vertical current source (see also figures $8(\mathrm{c})$ and (d)).

As mentioned earlier, the analysis above was focused on the equivalent current in the vertical section that is along the path from the source to the observation point. Since the distributions of the lightning-generated field and current are axisymmetric and the radius of the Fresnel zone in the transverse direction is small, the current distribution in the Fresnel zone can be approximately regarded as uniform in the transverse direction. Therefore, the analysis presented above is sufficient to illustrate the importance of the horizontal equivalent current regarding the sky waves at short observation distances and the importance of the vertical equivalent current regarding the sky waves at long observation distances.

Figure 10 summarizes the explanation of the polarity reversal of the sky wave's initial peak with the increase in the observation distance. Let us discuss the vertical electric field radiated by a negative return stroke. The vertical electric field component of the ground wave is negative (see Figure 10a). The skyward radiated electric field is composed of a positive horizontal component and a negative vertical component (see Figure 10b). The induced horizontal current density in the ionosphere is positive and the vertical current density is negative (see Figure 10c). At short observation distances, within $200 \mathrm{~km}$, the sky wave is dominated by the component generated by the horizontal equivalent current and, thus, the initial polarity of the sky wave is the same as that of the ground wave (see Figure 10d). However, when the observation distance is larger than $\sim 300 \mathrm{~km}$, the sky wave is dominated by the component generated by the vertical equivalent current in the ionosphere, resulting in a positive initial peak of the sky wave that is opposite to the groundwave polarity. The same explanations can also be readily applied to the lightning-radiated azimuthal magnetic field.

\subsection{Sky wave under nighttime ionosphere conditions}

The lightning-radiated EM wave reflection height in the nighttime $(\sim 85 \mathrm{~km})$ is higher than that in the daytime. The electron gyrofrequency is much larger than the electron-neutral collision rate for much of the reflection region (Jacobson et al., 2009). The lower ionosphere becomes an anisotropic medium in this situation. Therefore, the waveform of the sky wave will be different when the lightning-radiated EM wave propagates in different directions (e.g. Jacobson et al., 2009, 2012; Qin et al., 2017). In the following, we will test the validity of our proposed explanation taking into account the effect of the Earth's geomagnetic field $\left(\mathbf{B}_{\mathbf{E}}\right)$. The geomagnetic field is assumed to be $5 \times 10^{-5} \mathrm{~T}$. The geomagnetic inclination is set to $45^{\circ}$. 
In addition, positive ions and negative ions in the space are also taken into account in this case.

Figure 11 shows the calculated induced current density in the ionosphere for typical nighttime ionosphere conditions, and the LEMP is assumed to propagate from West to East (eastward propagation) in this case. The angle $\phi$ between the horizontal component of $\mathbf{B}_{\mathbf{E}}$ and the wave propagation direction equals $90^{\circ}$. The current density is the total current density produced by the electrons, positive ions and negative ions. Actually, the current produced by ions is much smaller than that produced by electrons. Figure 12 shows the sky wave calculated on the ground surface at different observation distances ranging from $100 \mathrm{~km}$ to $400 \mathrm{~km}$ under the same conditions. Comparing with the results without considering the Earth's geomagnetic field (see the Supporting Information), it can be seen that the horizontal current below $85 \mathrm{~km}$ diminishes considerably and the vertical current at $90 \mathrm{~km}$ increases appreciably after considering the geomagnetic field and assuming the eastward propagation of the LEMP. Therefore, at a larger observation distance (see figures $12 \mathrm{c}$ and d), the sky wave is more significantly dominated by the component generated by the vertical current in the Fresnel zone, compared with the results without considering the geomagnetic field. The initial polarity of the sky wave reverses from negative to positive similarly to the results without considering the geomagnetic field. The polarity reversal distance is $\sim 200 \mathrm{~km}$ under this condition. The analysis and explanation presented in section 3.3 are also valid for this complex case.

Figure 13 shows the calculated induced current density in the ionosphere for typical nighttime ionosphere conditions, and the LEMP is assumed to propagate from East to West (westward propagation). Figure 14 shows the sky wave calculated on the ground surface. Comparing with the results without considering the Earth's geomagnetic field (see the Supporting Information), it can be seen that the horizontal current density at $90 \mathrm{~km}$ greatly increases (see Figure 13d). This leads to a larger contribution of the horizontal current source to the sky wave. As the observation distance increases, the contribution of the vertical current source to the first sky wave increases in the same way as in the analysis in section 3.3. At observation distances larger than $300 \mathrm{~km}$ (see figures 14c and d), both sky-wave components, generated by the vertical current source and the horizontal current source, contribute to the first sky wave significantly. The superposition of these two components produces the doublepeak sky wave. It is worth noting that these two sky-wave components have only "one peak", similar to the situation without considering the geomagnetic field. This further validates the self-consistency of our explanation.

\section{Summary and discussion}

In this paper, we studied the phenomenon of the polarity reversal of the lightninggenerated sky wave using a time-domain method combining the FDTD method and the superposition principle of electromagnetic waves. In the proposed method, we calculated first the lightning-generated induced current in the ionosphere using the FDTD method. This current was then taken as a new source and used to simulate the sky waves at observation distances ranging from 100 to $400 \mathrm{~km}$. The horizontal and vertical components of the induced current density at different points in the ionosphere were presented and discussed. In addition, the contributions of the horizontal and vertical current sources to the sky waves were analyzed in detail. Furthermore, we proposed an explanation for the polarity reversal of the sky wave with the increase in the observation distance.

At short observation distances (within $200 \mathrm{~km}$ ), the first sky wave is dominated by the component generated by the horizontal current in the Fresnel zone. For longer observation 
distances (greater than $\sim 300 \mathrm{~km}$ ), the first sky wave is dominated by the component generated by the vertical current in the Fresnel zone. Since the sky wave components generated by the vertical current source and horizontal current source have opposite polarities, the polarity of the sky wave will reverse as the observation distance increases. This explanation is valid for both daytime and nighttime conditions. In addition, when the LEMP propagates from East to West under nighttime conditions, the horizontal current density at 90 $\mathrm{km}$ increases due to the presence of the geomagnetic field. At observation distances larger than $300 \mathrm{~km}$, both the sky-wave components generated by the vertical current source and the horizontal current source significantly contribute to the first sky wave. The superposition of these two components produces the double-peak sky wave.

In this paper, we mainly focused on the relationship between the sky wave and the induced current in the ionosphere. However, it is still unclear how the electron moves in the presence of the geomagnetic field when the LEMP propagates in different directions. In addition, the decomposition of the induced current in the ionosphere into equivalent horizontal and vertical components does not mean that the current is strictly constrained in these two directions. These two components are equivalent currents in the horizontal and vertical directions. In view of the above, the explanation provided in the paper to the polarity reversal of the sky wave cannot be considered as a comprehensive physical description, but is convergent to an engineering explanation. However, this is the first explanation in the time domain for the polarity reversal of the sky wave, and it gives us some new insights into the reflection mechanisms from the lower ionosphere.

Finally, although our new proposed method was used to study the lightning-generated sky wave in this paper, we believe this method can also be used to analyze other reflection and scattering problems of EM waves.

\section{Acknowledgments}

This work was supported in part by the National Key R\&D Program of China under Grant 2017YFC1501505, the National Natural Science Foundation of China under Grant 41775006 and Grant 41675006, the scholarship from the China Scholarship Council, the Swiss National Science Foundation under Project No. 200020_175594, and the European Union's Horizon 2020 research and innovation program under grant agreement No. 737033LLR. All data presented in this paper is available at DOI:10.6084/m9.figshare.11566749.

\section{Appendix A: Validation of Our FDTD Codes}

In order to validate our FDTD codes, we compared our simulated results with the measured waveforms published by Qin et al. (2017). Figure A1 shows the vertical electric field at an observation distance of $189 \mathrm{~km}$ under nighttime conditions. The black line is the measured data adapted from Figure 8b of Qin et al. (2017), and the red line is the result calculated using our FDTD codes. We used the lightning source presented in Equation (12) of Qin et al. (2017). The grid size was set to $100 \mathrm{~m}$. In addition, the effect of the geomagnetic field was considered. It can be clearly seen that our simulated result is in good agreement with the measured waveform, thus validating our FDTD codes. Our simulated sky wave is a little larger than the measured data. This can be attributed to the difference between the input source and the actual lightning source and the numerical dispersion of FDTD. The latter slightly reduces the peak of the ground wave. However, this does not have any influence on the results and analyses in our paper. 


\section{References}

Azadifar, M., Li, D., Rachidi, F., Rubinstein, M., Diendorfer, G., Schulz, W., et al. (2017).

Analysis of lightning-ionosphere interaction using simultaneous records of source current and $380 \mathrm{~km}$ distant electric field. Journal of Atmospheric and Solar-Terrestrial Physics, 159, 4856. https://doi.org/10.1016/j.jastp.2017.05.010

Berenger, J.-P. (2005). Long Range Propagation of Lightning Pulses Using the FDTD Method. IEEE Transactions on Electromagnetic Compatibility, 47(4), 1008-1012. https://doi.org/10.1109/temc.2005.858747

Bickel, J. E., Ferguson, J. A., \& Stanley, G. V. (1970). Experimental Observation of Magnetic Field Effects on VLF Propagation at Night. Radio Science, 5(1), 19-25. https://doi.org/10.1029/rs005i001p00019

Budden, K. G. (1988), The Propagation of Radio Waves: The Theory of Radio Waves of Low Power in the Ionosphere and Magnetosphere, Cambridge Univ. Press, Cambridge.

Cheng, Z., \& Cummer, S. A. (2005). Broadband VLF measurements of lightning-induced ionospheric perturbations. Geophysical Research Letters, 32, L08804. https://doi.org/10.1029/2004GL022187

Cummer, S. A. (1997). An analysis of new and existing FDTD methods for isotropic cold plasma and a method for improving their accuracy. IEEE Transactions on Antennas and Propagation, 45(3), 392-400. https://doi.org/10.1109/8.558654

Cummer, S. A. (2003), A simple, nearly perfectly matched layer for general electromagnetic media. IEEE Microwave and Wireless Components Letters, 13(3), 128-130.

https://doi.org/10.1109//mwc.2003.810124

Cummer, S. A., \& Inan, U. S. (2000). Modeling ELF radio atmospheric propagation and extracting lightning currents from ELF observations. Radio Science, 35(2), 385-394. https://doi.org/10.1029/1999rs002184

Cummer, S. A., Inan, U. S., \& Bell, T. F. (1998). IonosphericDregion remote sensing using VLF radio atmospherics. Radio Science, 33(6), 1781-1792.

https://doi.org/10.1029/98rs02381

Ferguson, J. A., Snyder, F. P., Morfitt, D. G., \& Shellman, C. H. (1989). Long-wave propagation capability and documentation (Tech. Doc.), Naval Ocean Systems Center, San Diego, CA.

Haddad, M. A., V. A. Rakov, and S. A. Cummer (2012), New measurements of lightning electric fields in Florida: Waveform characteristics, interaction with the ionosphere, and peak current estimates, J. Geophys. Res., 117, D10101, https://doi.org/10.1029/2011JD017196.

Han, F., and S. A. Cummer (2010a), Midlatitude daytime $D$ region ionosphere variations measured from radio atmospherics, Journal of Geophysical Research, 115, A10314, https://doi.org/10.1029/2010JA015715.

Han, F., and S. A. Cummer (2010b), Midlatitude nighttime $D$ region ionosphere variability on hourly to monthly time scales, Journal of Geophysical Research, 115, A09323, https://doi.org/10.1029/2010JA015437

Hu, W., \& Cummer, S. A. (2006). An FDTD model for low and high altitude lightninggenerated EM fields. IEEE Transactions on Antennas and Propagation, 54, 15131522. https://doi.org/10.1109/TAP.2006.87433 
Jacobson, A. R., Shao, X.-M., \& Holzworth, R. (2009). Full-wave reflection of lightning long-wave radio pulses from the ionospheric D region: Numerical model. Journal of Geophysical Research, 114, A03303. https://doi.org/10.1029/2008JA013642

Jacobson, A. R., X.-M. Shao, and E. Lay (2012), Time domain waveform and azimuth variation of ionospherically reflected VLF/LF radio emissions from lightning, Radio Sci., 47, RS4001. https://doi.org/10.1029/2012RS004980

Lay, E. H., \& Shao, X.-M. (2011). High temporal and spatial-resolution detection of D-layer fluctuations by using time-domain lightning waveforms. Journal of Geophysical Research, 116, A01317. https://doi.org/10.1029/2010ja016018

Li, J., S. A. Cummer, W. A. Lyons, and T. E. Nelson (2008), Coordinated analysis of delayed sprites with high-speed images and remote electromagnetic fields, J. Geophys. Res., 113, D20206, https://doi.org/10.1029/2008JD010008.

Maloney, J. G., \& Smith, G. S. (1992). The use of surface impedance concepts in the finitedifference time-domain method. IEEE Transactions on Antennas and Propagation, 40(1), 38-48. https://doi.org/10.1109/8.123351

Marshall, R. A. (2012). An improved model of the lightning electromagnetic field interaction with the D-region ionosphere. Journal of Geophysical Research, 117, A03316. https://doi.org/10.1029/2011JA017408

Morfitt, D. G., \& Shellman, C. H.(1976). MODESRCH: An improved computer program for obtaining ELF/VLF/LF mode constants in an Earth-ionosphere waveguide, Nav. Electron.

Lab. Cent., San Diego, Calif. https://apps.dtic.mil/dtic/tr/fulltext/u2/a032573.pdf

Mostajabi, A., Li, D., Azadifar, M., Rachidi, F., Rubinstein, M., Diendorfer, G., et al. (2019). Analysis of a bipolar upward lightning flash based on simultaneous records of currents and 380-km distant electric fields. Electric Power Systems Research, 174, 105845. https://doi.org/10.1016/j.epsr.2019.04.023

Qin, Z., Chen, M., Zhu, B., \& Du, Y. (2017). An improved ray theory and transfer matrix method-based model for lightning electromagnetic pulses propagating in Earth-ionosphere waveguide and its applications. Journal of Geophysical Research: Atmospheres, 122, 712727. https://doi.org/10.1002/2016JD025599

Ratcliffe, J. A. (1959), The magneto-ionic theory and its applications to the ionosphere, Cambridge University Press, Cambridge.

Said, R. K. (2009). Accurate and efficient long-range lightning geo-location using a VLF radio atmospheric waveform bank. Stanford University.

Shao, X.-M., Lay, E. H., \& Jacobson, A. R. (2013). Reduction of electron density in the night-time lower ionosphere in response to a thunderstorm. Nature Geoscience, 6, 29-33. https://doi.org/10.1038/ngeo1668

Smith, D. A., Heavner, M. J., Jacobson, A. R., Shao, X. M., Massey, R. S., Sheldon, R. J., \& Wiens, K. C. (2004). A method for determining intracloud lightning and ionospheric heights from VLF/LF electric field records. Radio Science, 39, RS1010.

https://doi.org/10.1029/2002rs002790

Somu, V. B., Rakov, V. A., Haddad, M. A., \& Cummer, S. A. (2015). A study of changes in apparent ionospheric reflection height within individual lightning flashes. Journal of Atmospheric and Solar-Terrestrial Physics, 136, 66-79.

https://doi.org/10.1016/j.jastp.2015.09.007 
Thomson, N. R. (1993). Experimental daytime VLF ionospheric parameters. Journal of Atmospheric and Terrestrial Physics, 55(2), 173-184. https://doi.org/10.1016/00219169(93)90122-F

Tran, T. H., Baba, Y., Somu, V. B., \& Rakov, V. A. (2017). FDTD modeling of LEMP propagation in the Earth-ionosphere waveguide with emphasis on realistic representation of lightning source. Journal of Geophysical Research: Atmospheres, 122, 12,918-12,937. https://doi.org/10.1002/2017JD027305

Uman, M. A., McLain, D. K., \& Krider, E. P. (1975). The electromagnetic radiation from a finite antenna. American Journal of Physics, 43(1), 33-38. https://doi.org/10.1119/1.10027

Volland, H. (1995), Longwave sferics propagation within the atmospheric waveguide, in Atmospheric Electrodynamics, vol. 2, pp. 65-93, CRC Press, Boca Raton, Fla.

Wait, J. R., \& Spies, K. P. (1964). Characteristics of the Earth-ionosphere waveguide for VLF radio waves (NBS Tech. Note 300), National Bureau of Standards, Boulder, CO. https://nvlpubs.nist.gov/nistpubs/Legacy/TN/nbstechnicalnote300.pdf

Wait, J. R., \& Walters, L. C. (1963). Reflection of VLF radio waves from an inhomogeneous ionosphere. Part I. Exponentially varying isotropic model. J. Res. NBS D, 67, 361-367. https://nvlpubs.nist.gov/nistpubs/jres/67D/jresv67Dn3p361_A1b.pdf

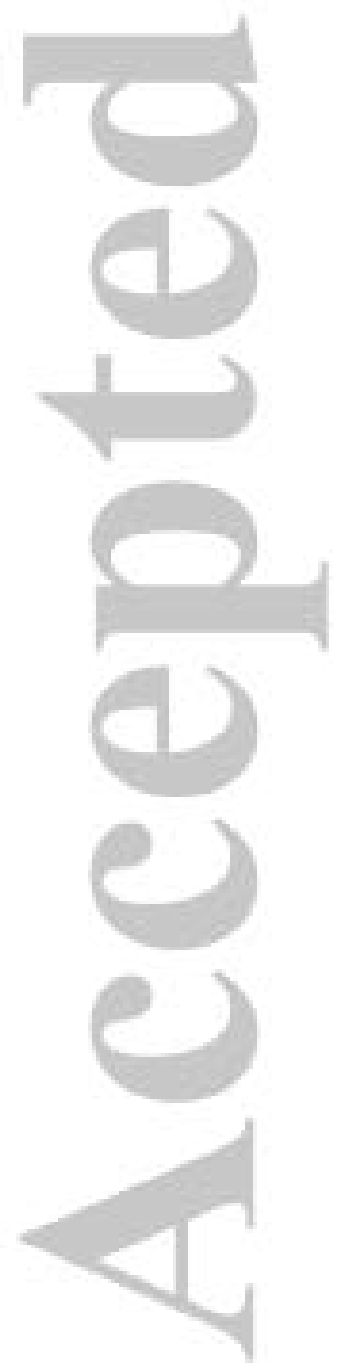


(a)

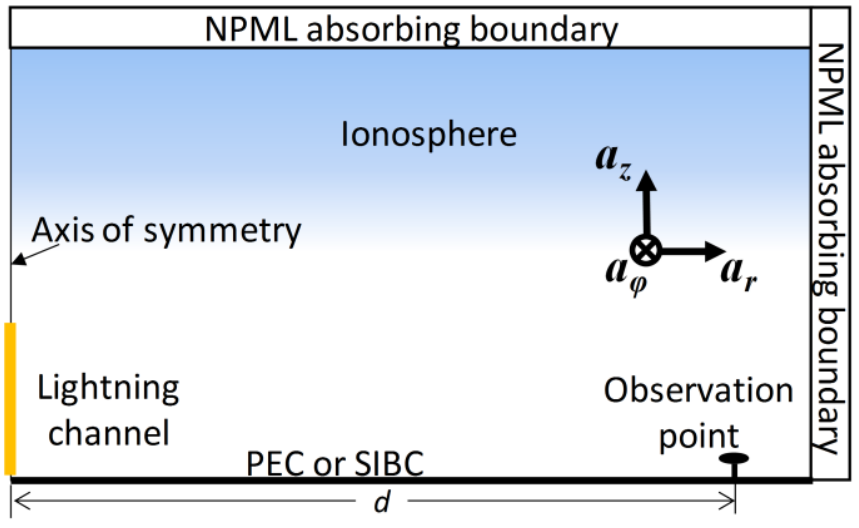

(b)

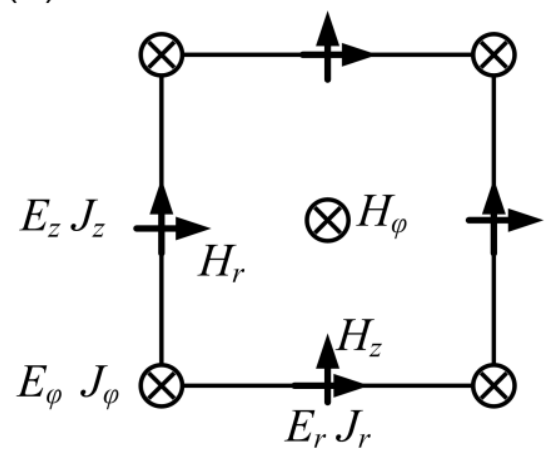

Figure 1. (a) Geometry of the adopted 2-D FDTD model and the vector direction definition in this paper. (b) Mesh scheme of the FDTD model. $\mathbf{J}$ and $\mathbf{E}$ are spatially collocated. 


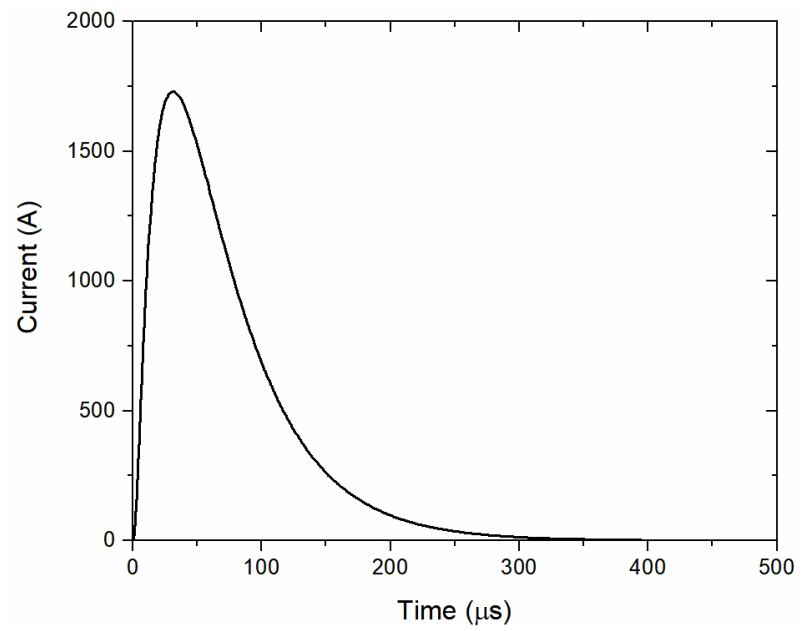

Figure 2. Current along the lightning discharge channel. 


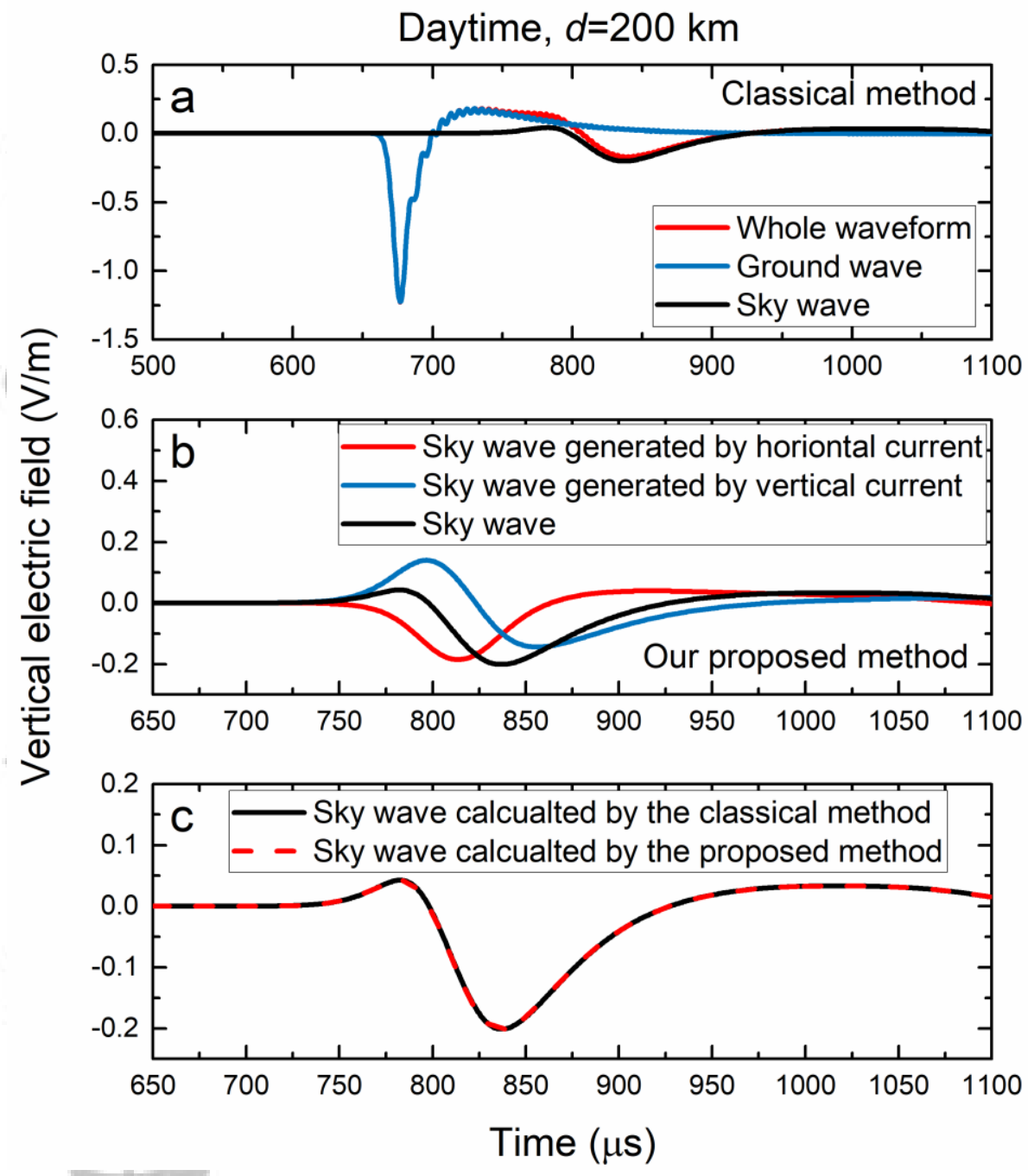

Figure 3. Comparison of sky waves calculated using (a) the classical method, (b) the proposed method, and (c) comparison of the sky waves for both methods. The calculations were made for daytime ionosphere conditions at an observation distance of $200 \mathrm{~km}$. The observation point is located at ground level. The ground conductivity is $0.005 \mathrm{~S} / \mathrm{m}$. 


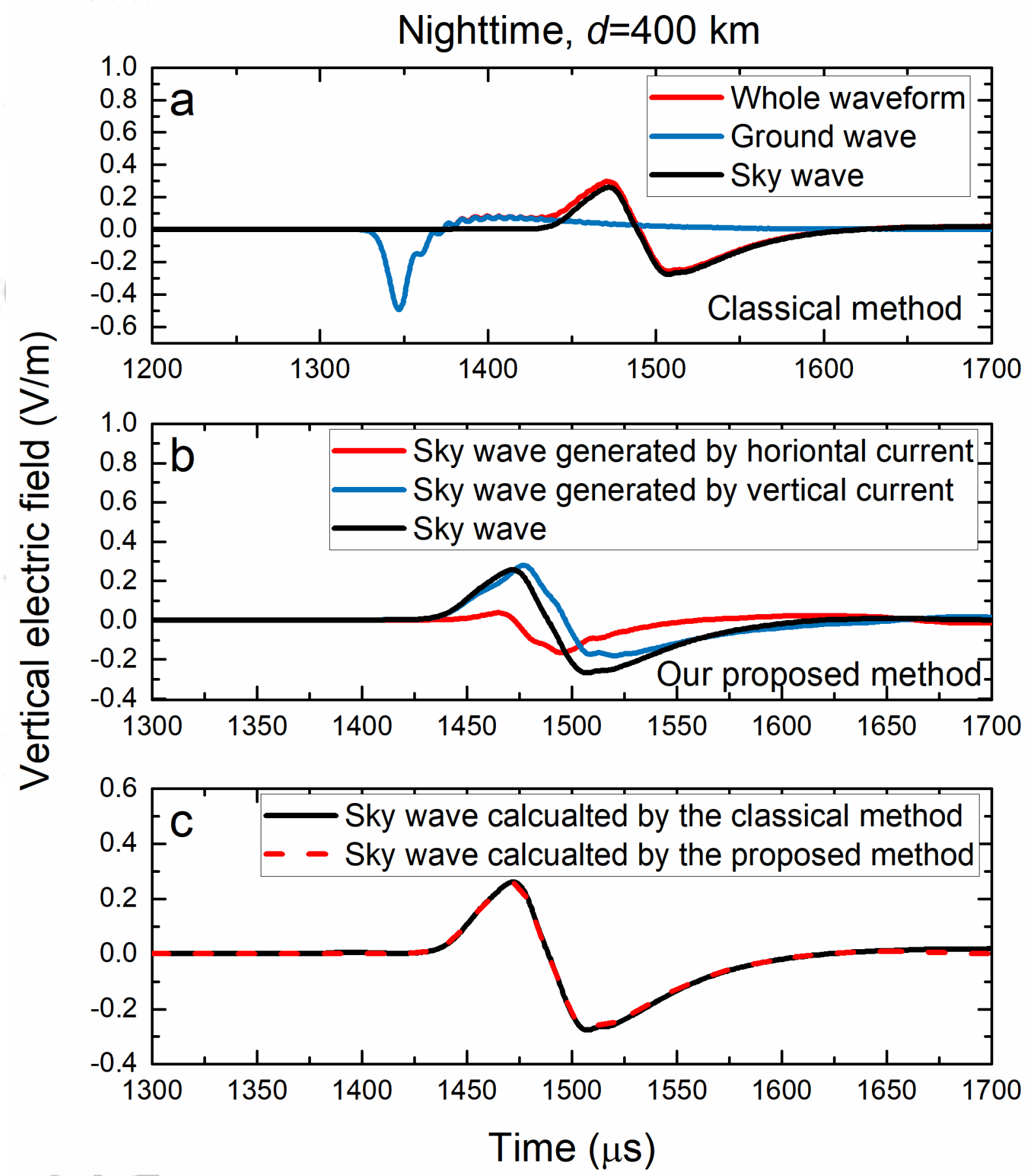

Figure 4. Comparison of sky waves calculated using (a) the classical method, (b) the proposed method, and (c) comparison of the sky waves for both methods. The calculations were made for nighttime ionosphere conditions at an observation distance of $400 \mathrm{~km}$. 

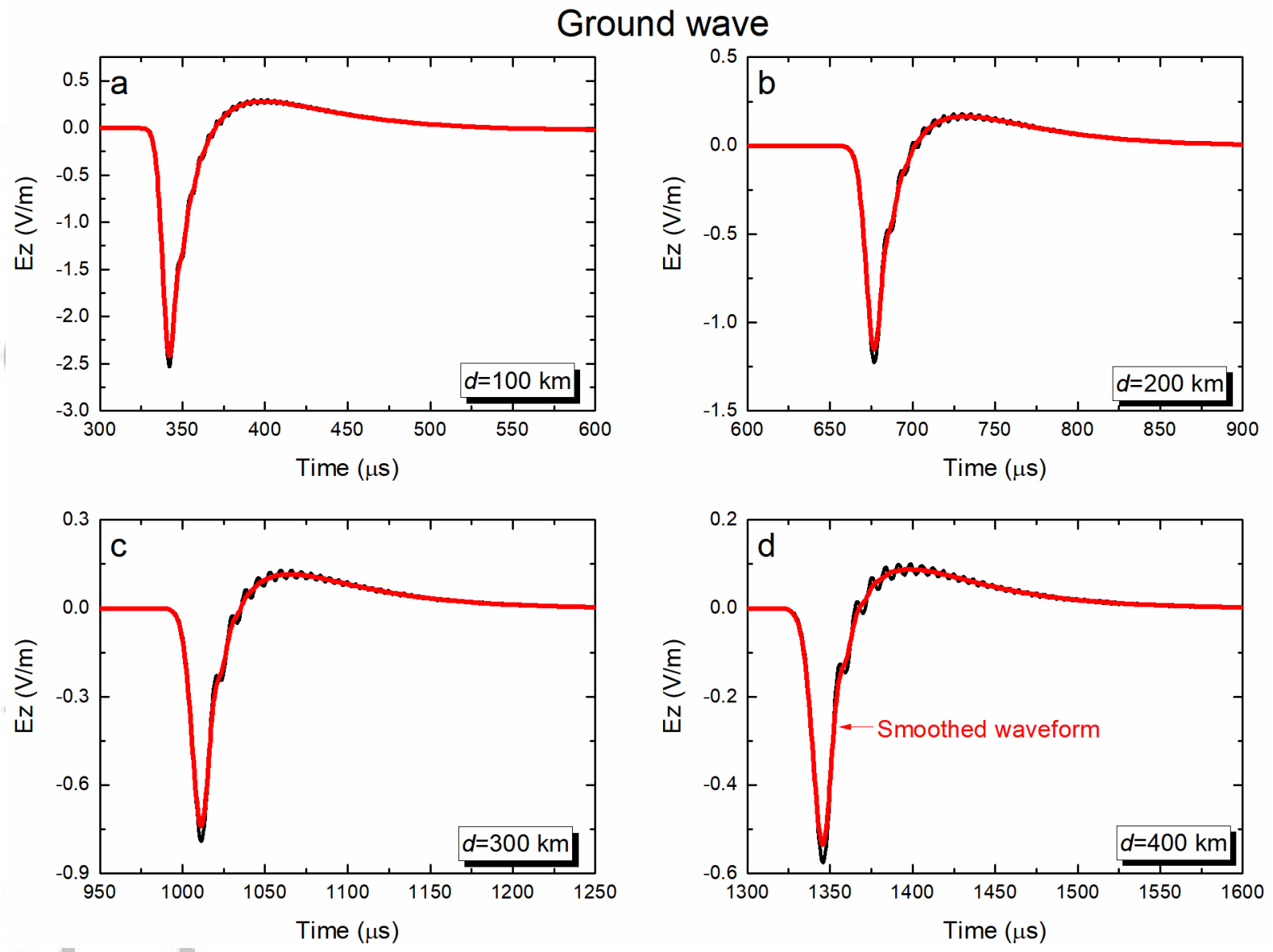

Figure 5. The ground wave calculated on the ground surface at different observation distances: (a) $100 \mathrm{~km}$, (b) $200 \mathrm{~km}$, (c) $300 \mathrm{~km}$ and (d) $400 \mathrm{~km}$. The small oscillation of the waveform is caused by the numerical dispersion. The red lines are the smoothed waveforms. 


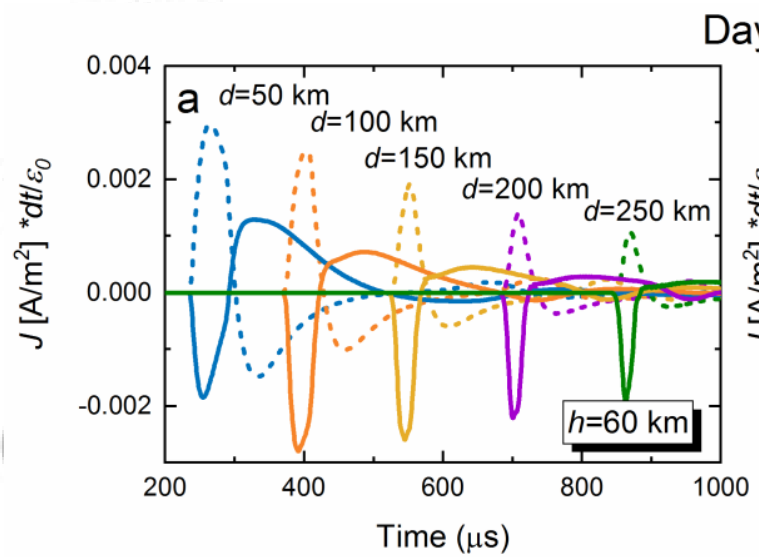

Daytime
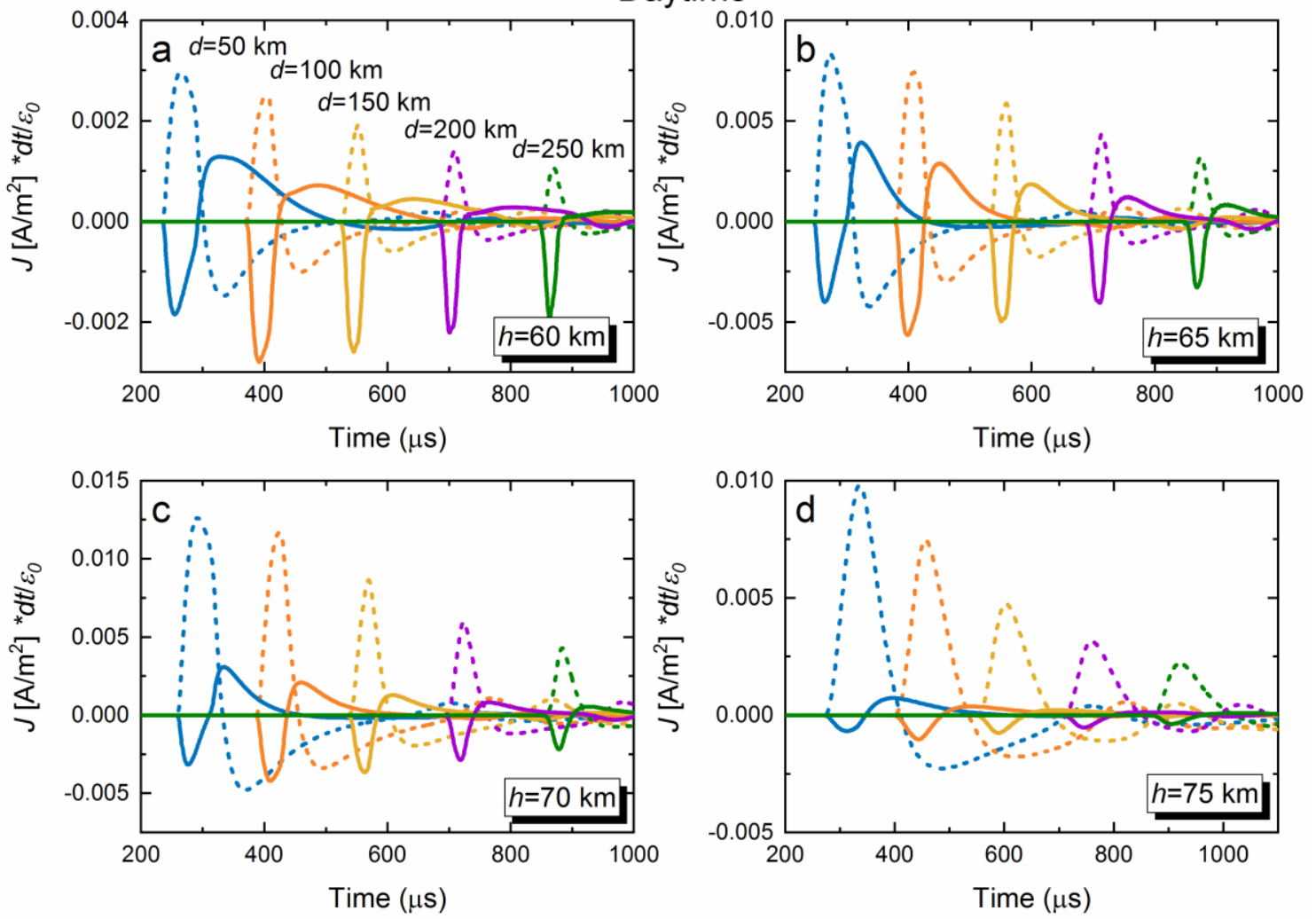

Dashed lines: horizontal current density

Solid lines: vertical current density

Figure 6. The scaled induced current density in the ionosphere at four different heights, from $h=60 \mathrm{~km}$ to $h=75 \mathrm{~km}$, and five horizontal distances, from $d=50 \mathrm{~km}$ to $d=250 \mathrm{~km}$, for daytime ionosphere conditions. The dashed lines and solid lines represent the horizontal current density and the vertical current density, respectively. The different line colors represent different horizontal distances (identified in the upper left plot). The time step $\Delta t$ is $0.5 \mu \mathrm{s}$. The vector direction definition is shown in Figure 1(a). 


\section{Daytime}
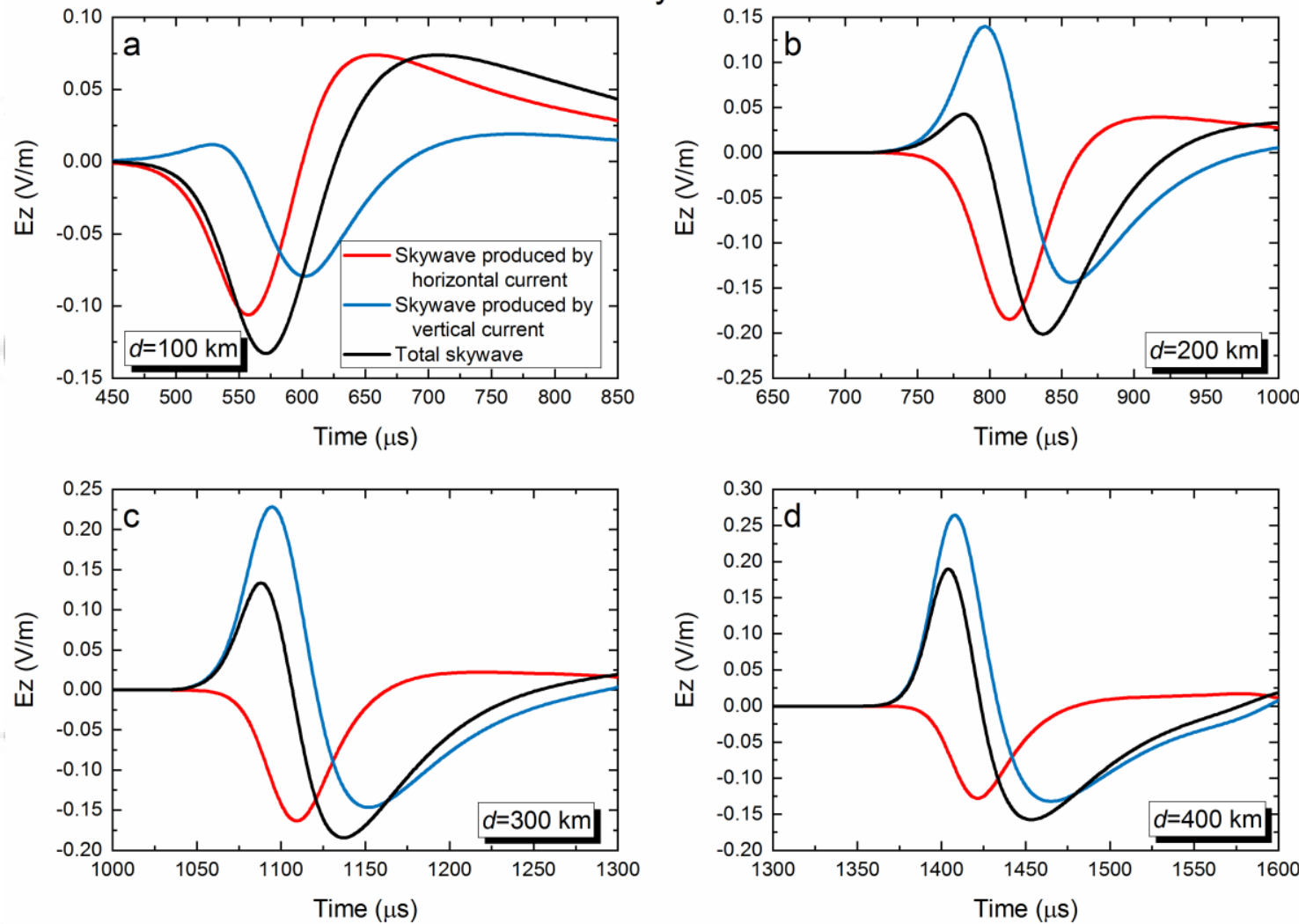

Figure 7. The sky wave calculated on the ground surface at different observation distances: (a) $100 \mathrm{~km}$, (b) $200 \mathrm{~km}$, (c) $300 \mathrm{~km}$ and (d) $400 \mathrm{~km}$. The red lines show the sky wave component generated by the horizontal currents in the ionosphere. The blue lines show the sky wave component generated by the vertical currents in the ionosphere. The black lines are the summation of these two components that is the whole first sky wave. The current density in the entire ionosphere is considered in this calculation. 

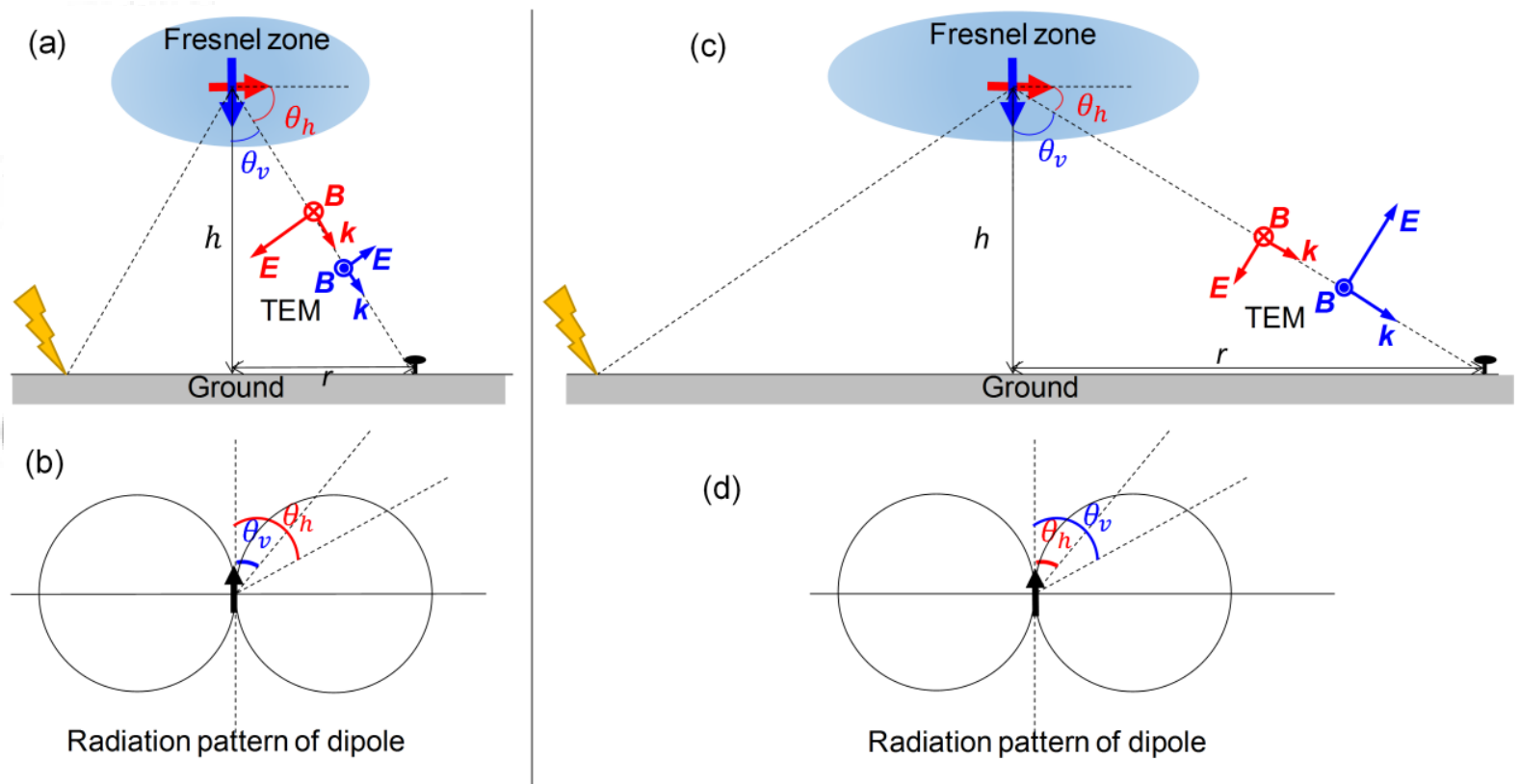

(d)

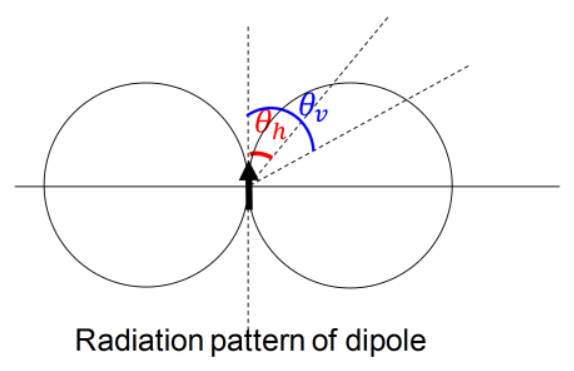

Figure 8. Illustration of the contributions of a horizontal dipole and a vertical dipole in the Fresnel zone to the sky wave at ground level. Assume these two dipoles have the same current. $(a, b)$ At short distance, the zenith angle of the horizontal dipole $\left(\theta_{h}\right)$ is larger than that of the vertical dipole $\left(\theta_{v}\right)$ at the same position. According to the radiation pattern of the dipole, the radiation of the horizontal dipole will be larger than that of the vertical dipole. (c, d) For large observation distances, the radiation from the vertical dipole dominates. 

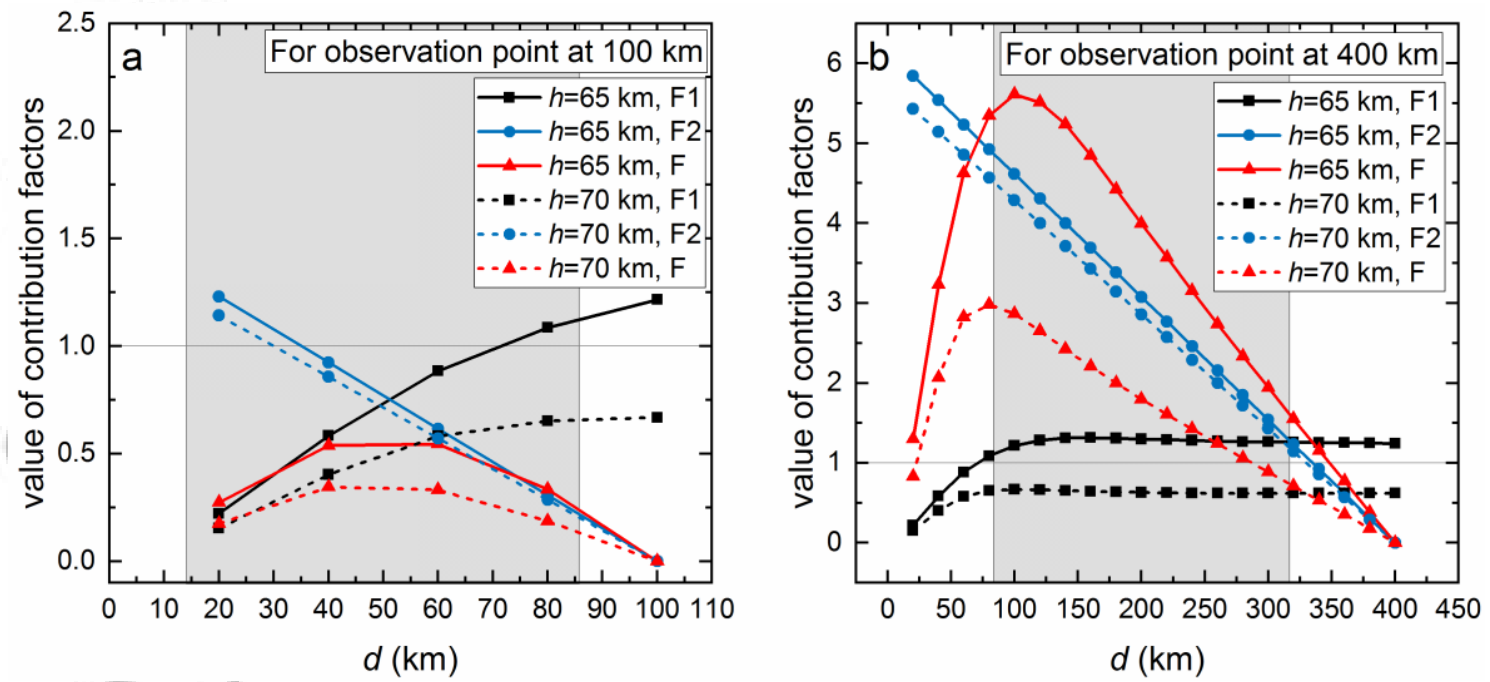

Figure 9. Calculated contribution factors $F$ (red lines), $F_{1}$ (black lines), and $F_{2}$ (blue lines) at different positions in the ionosphere. (a) and (b) are the results for the field observation points on the ground level at distances of $100 \mathrm{~km}$ and $400 \mathrm{~km}$, respectively. $d$ and $h$ are the horizontal distance and the height of the sampling point in the ionosphere, respectively. The shadow region marks the largest horizontal range of the Fresnel zone for an EM wave at 15 $\mathrm{kHz}$.

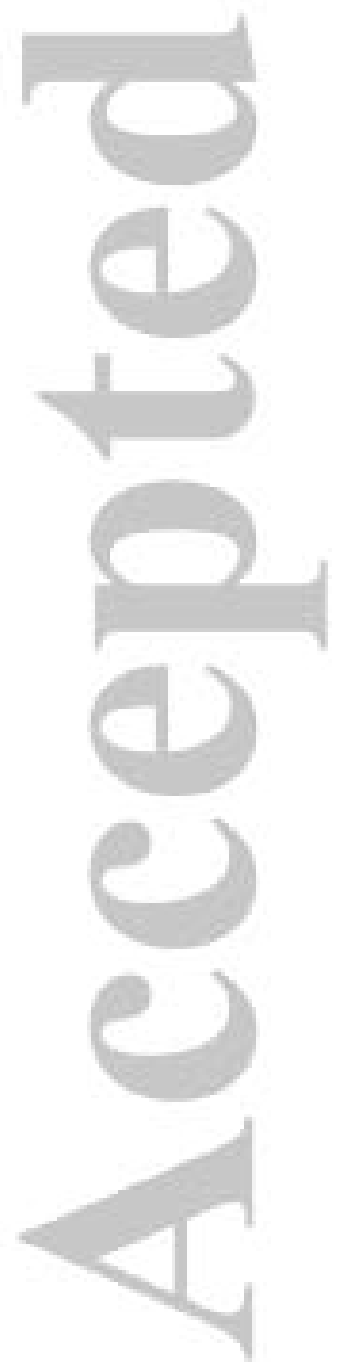


(c)

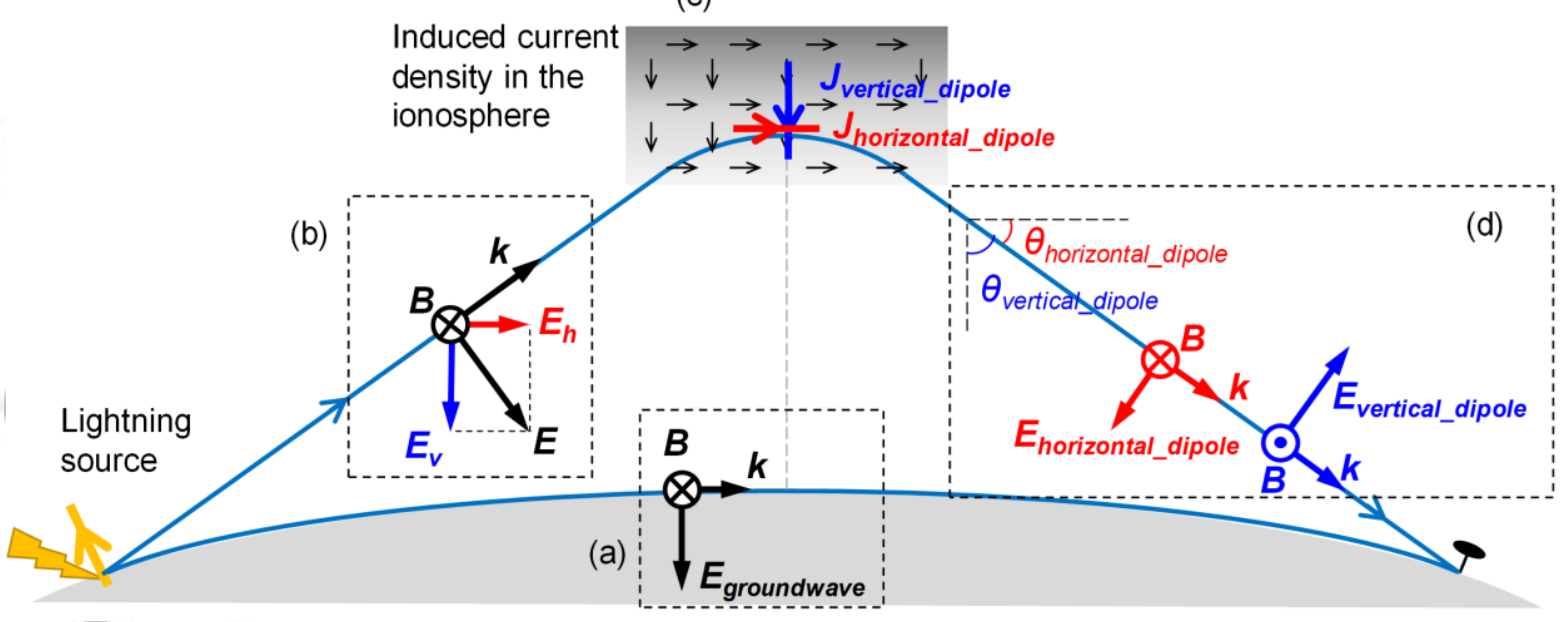

Figure 10. Schematic explanation of the polarity reversal of the first sky wave. (a) The propagation of the ground wave. (b) The lightning-radiated skyward wave. (c) The induced current density in the ionosphere. (d) The radiation of the induced current. See text for details. 
Nighttime, eastward propagation $\left(\phi=90^{\circ}\right)$
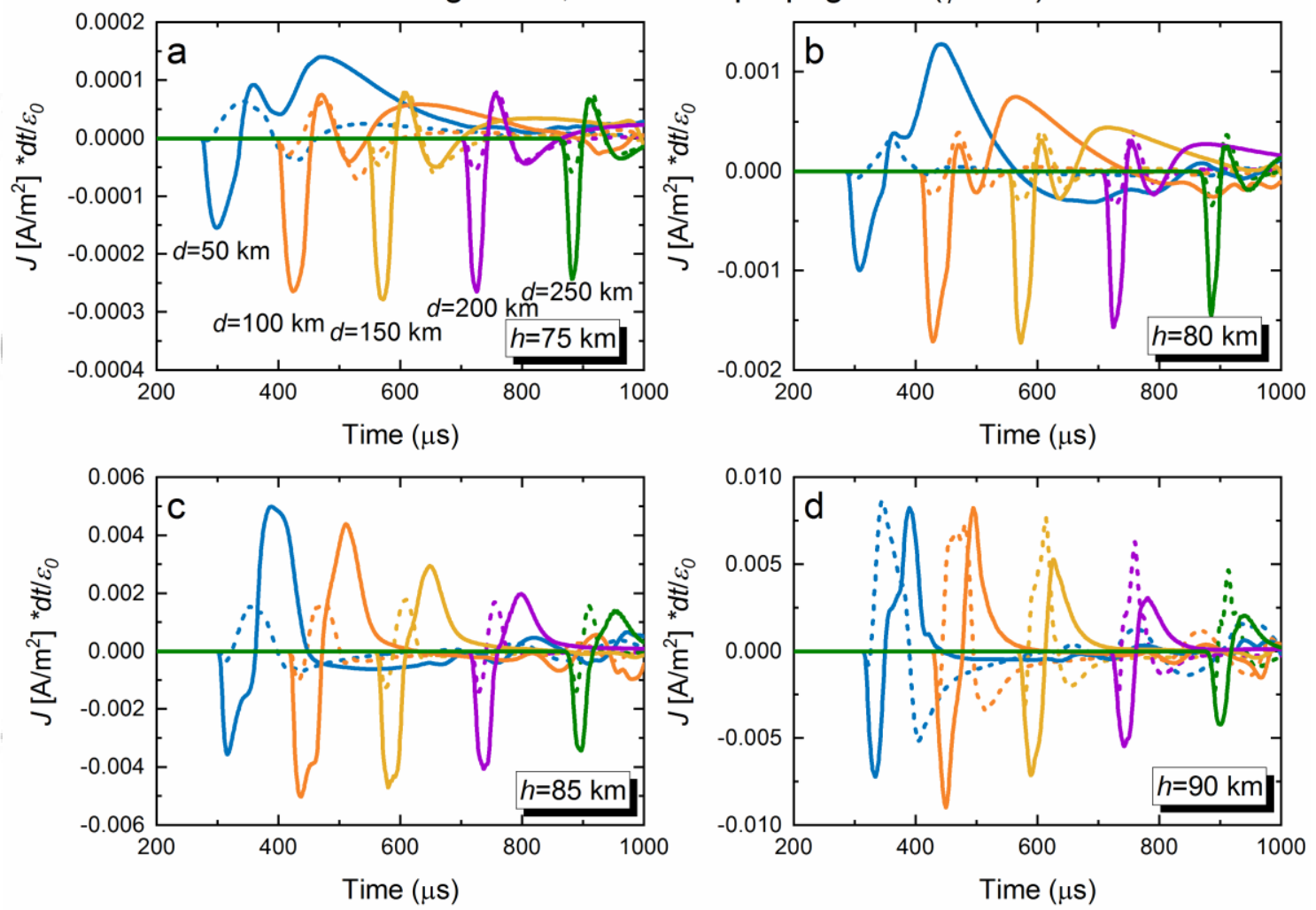

Dashed lines: horizontal current density

Solid lines: vertical current density

Figure 11. Similar to Figure 6, the scaled induced current density in the ionosphere at different heights $(h)$ and horizontal distances $(d)$ for an eastward propagation case under typical nighttime conditions. 
Nighttime, eastward propagation $\left(\phi=90^{\circ}\right)$
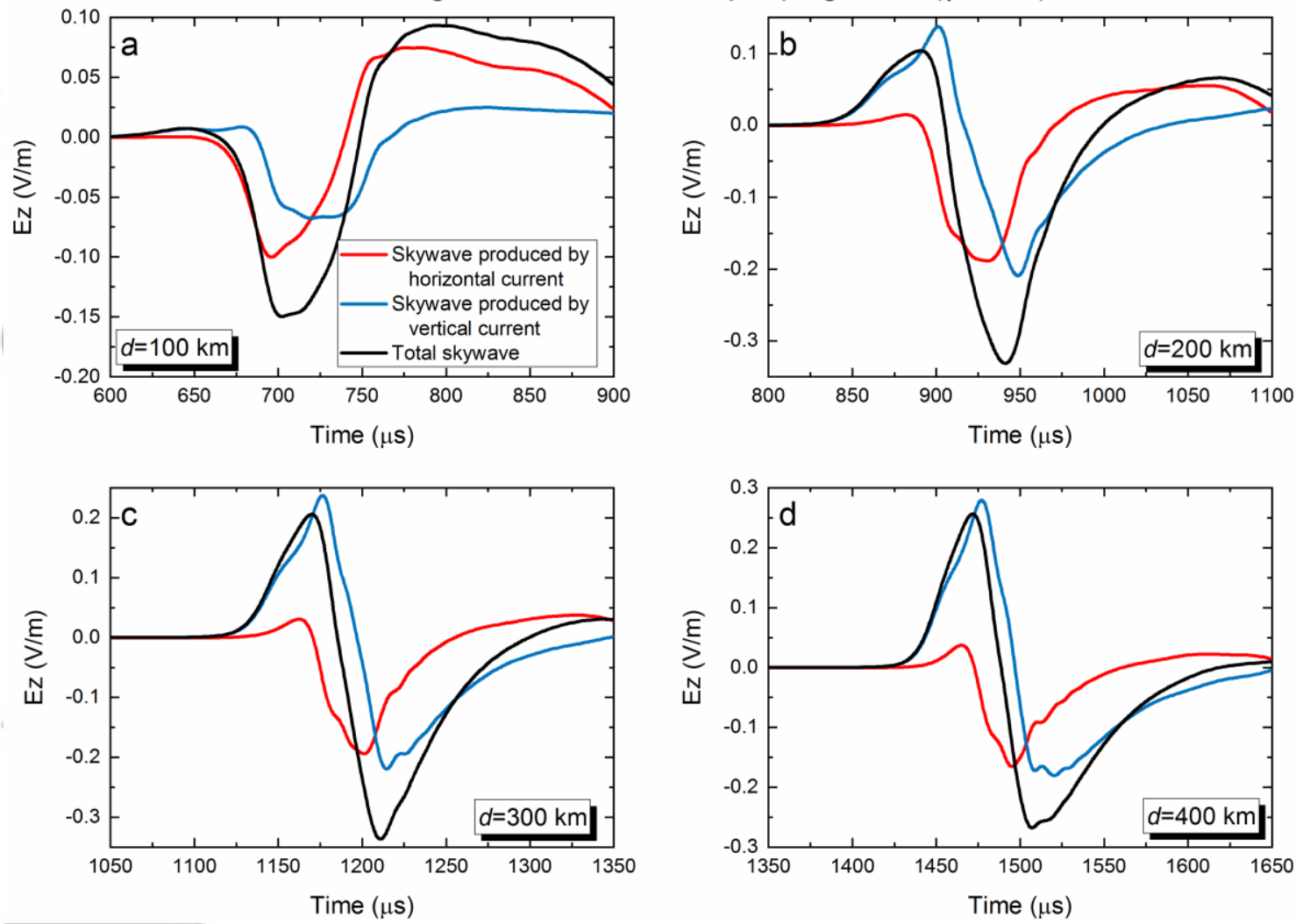

Figure 12. Similar to Figure 7, the sky wave calculated on the ground surface at different observation distances of (a) $100 \mathrm{~km}$, (b) $200 \mathrm{~km}$, (c) $300 \mathrm{~km}$ and (d) $400 \mathrm{~km}$ for an eastward propagation case under typical nighttime conditions. 
Nighttime, westward propagation $\left(\phi=270^{\circ}\right)$
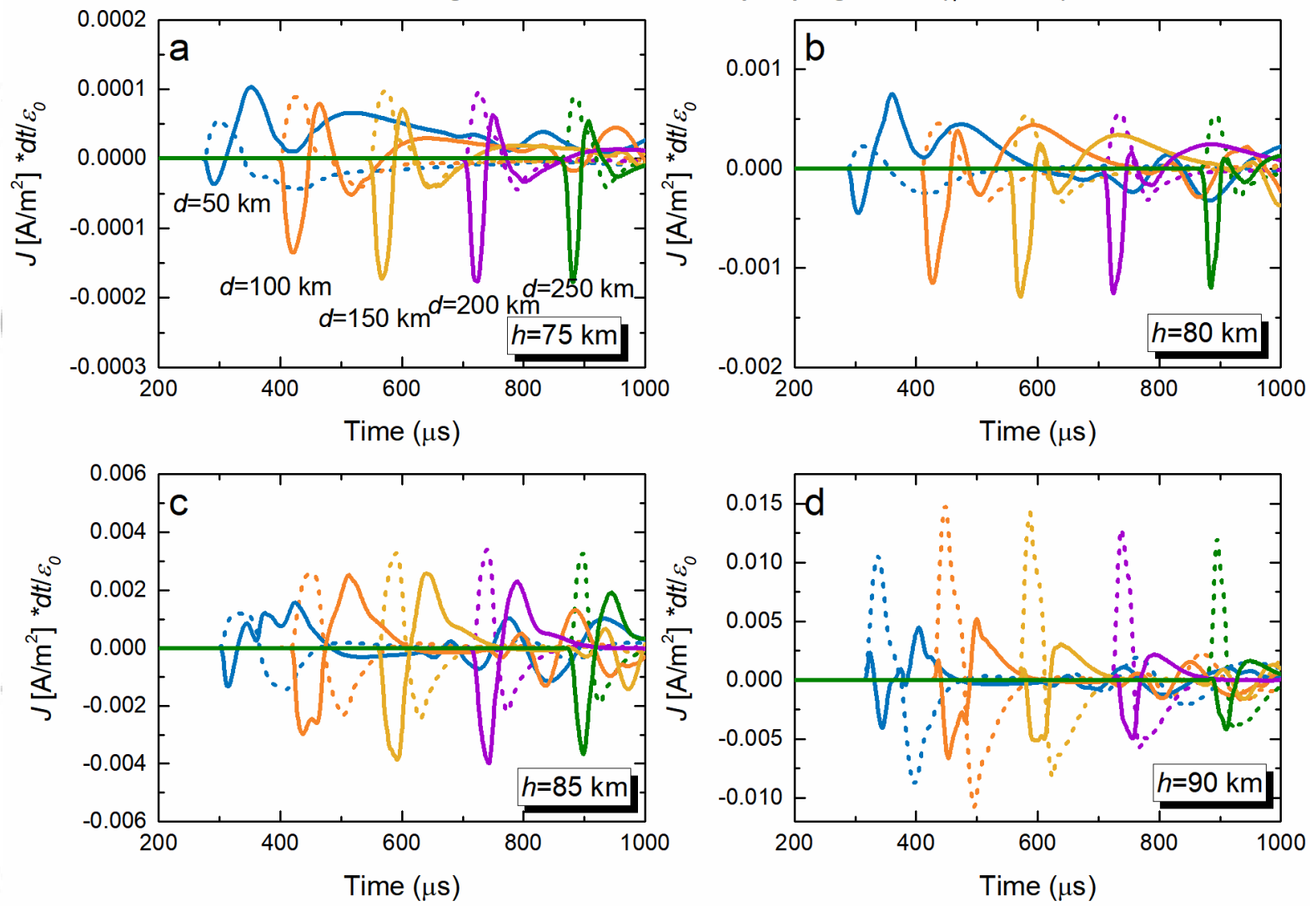

Dashed lines: horizontal current density

Solid lines: vertical current density

Figure 13. Similar to Figure 11, the scaled induced current density in the ionosphere at different heights $(h)$ and horizontal distances $(d)$ for a westward propagation case under typical nighttime conditions. 
Nighttime, westward propagation $\left(\phi=270^{\circ}\right)$
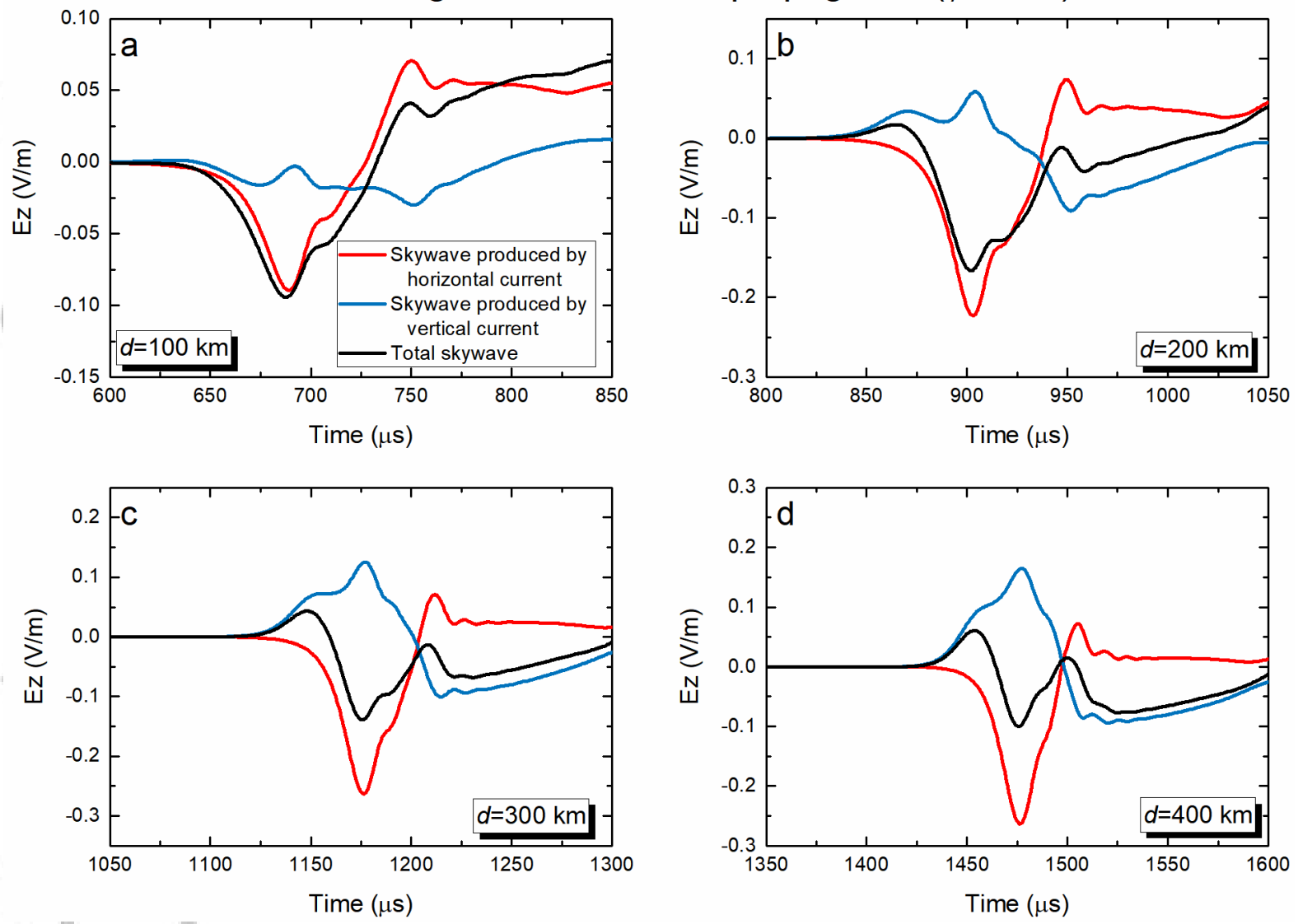

Figure 14. Similar to Figure 12, the sky wave calculated on the ground surface at different observation distances of (a) $100 \mathrm{~km}$, (b) $200 \mathrm{~km}$, (c) $300 \mathrm{~km}$ and (d) $400 \mathrm{~km}$ for a westward propagation case under typical nighttime conditions. 

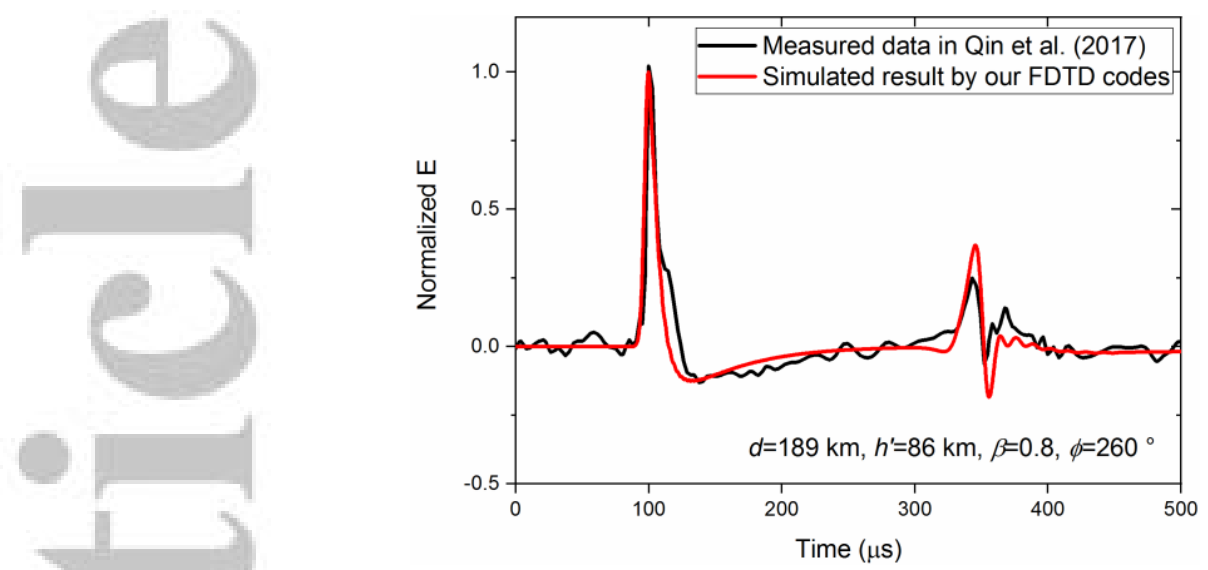

Figure A1. The normalized vertical electric field at an observation distance of $189 \mathrm{~km}$ under nighttime conditions $\left(h^{\prime}=86 \mathrm{~km}\right.$ and $\beta=0.8 \mathrm{~km}^{-1}$ ). The black line is the measured data adopted from Figure $8 \mathrm{~b}$ of Qin et al. (2017), and the red line is the result calculated using our FDTD codes.

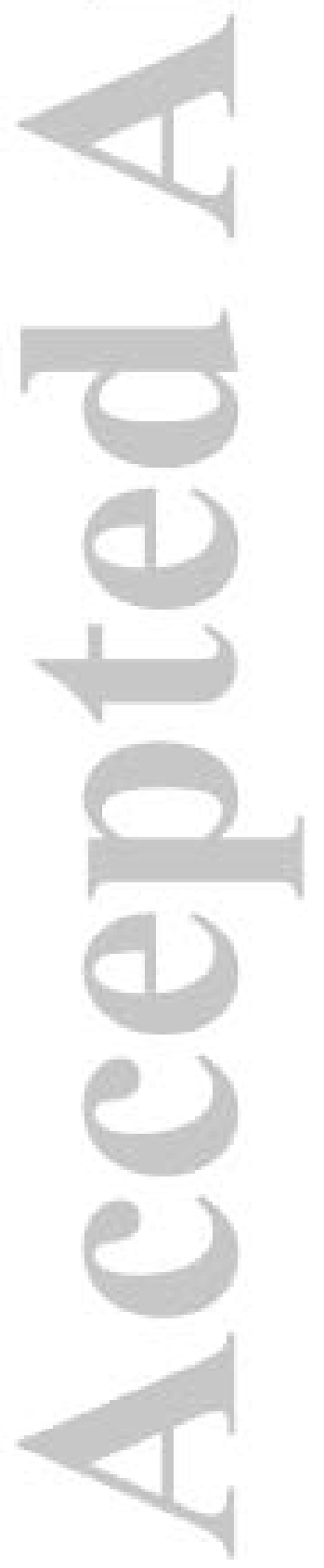

